\title{
A Study of the LoRa Signal Propagation in Forest, Urban, and Suburban Environments
}

\author{
Ana Elisa Ferreira - Fernando M. Ortiz • \\ Luís Henrique M. K. Costa • Brandon \\ Foubert · Ibrahim Amadou • Nathalie \\ Mitton
}

Received: date / Accepted: date

\begin{abstract}
Sensing is an activity of paramount importance for smart cities. The coverage of large areas based on reduced infrastructure and low energy consumption is desirable. In this context, Low Power Wide Area Networks (LPWAN) play an important role. In this paper, we investigate LoRa, a low power technology offering large coverage, but low transmission rates. Radio range and data rate are tunable by using different spreading factors and coding rates, which are configuration parameters of the LoRa PHY layer.

LoRa can cover large areas but variations in the environment affect link quality. This work studies the propagation of LoRa signals in forest, urban, and suburban vehicular environments. Besides being environments with variable propagation conditions, we evaluate scenarios with node mobility. To char-
\end{abstract}

\footnotetext{
A. Ferreira

Federal University of Rio de Janeiro - GTA/PEE/COPPE, Rio de Janeiro, Brazil

Federal Technology Education Center Celso Suckow da Fonseca - UneDPetrópolis/CEFET-

RJ, Petrópolis, Brazil

E-mail: ferreira@gta.ufrj.br

F. Ortiz

Federal University of Rio de Janeiro - GTA/PEE/COPPE, Rio de Janeiro, Brazil

E-mail: fmolano@gta.ufrj.br

L. Costa

Federal University of Rio de Janeiro - GTA/PEE/COPPE, Rio de Janeiro, Brazil

E-mail: luish@gta.ufrj.br

B. Foubert

National Institute for Research in Computer Science and Automatic Control, Lille, France E-mail: brandon.foubert@inria.fr

I. Amadou

National Institute for Research in Computer Science and Automatic Control, Lille, France E-mail: amadou68@gmail.com

N. Mitton

National Institute for Research in Computer Science and Automatic Control, Lille, France E-mail: nathalie.mitton@inria.fr
} 
acterize the communication link we mainly use the Received Signal Strength Indicator (RSSI), Signal to Noise Ratio (SNR), and Packet Delivery Ratio (PDR). As for node mobility, speeds are chosen according to prospective applications. Our results show that the link reaches up to $250 \mathrm{~m}$ in the forest scenario, while in the vehicular scenario it reaches up to $2 \mathrm{~km}$. In contrast, in scenarios with high-density buildings and human activity, the maximum range of the link reaches up $200 \mathrm{~m}$ in the urban scenario.

Keywords Smart cities - Low power wide area networks · Wireless sensor networks · LoRa technology

\section{Introduction}

New radio technologies for communication in the Internet of Things (IoT) and Wireless Sensors Network (WSN) simultaneously instigate and meet the demand for new applications, in new usage environments. New uses have brought the need for communication in environments as different as cities, rural, and natural areas. Electromagnetic propagation conditions depend on the environment and may be very dynamic. As a consequence, radio communication technologies based on various modulation techniques and using different frequency bands appear. Among them, Low Power Wide Area Networks (LPWAN) is a class of networks characterized by low power consumption and coverage of large areas, two performance metrics important to have green communications.

Wireless local and personal area networks such as IEEE 802.11, Zigbee or Bluetooth are frequently considered for smart city applications. Nonetheless, as compared to LPWAN, they usually provide larger transmission rates but a shorter range and less energy efficiency. LPWAN technologies promise minimal power consumption and long ranges, while allowing the reuse of frequencies of legacy communication networks. In this paper, we focus on LoRa (Long Range), a technology belonging to the set of LPWAN solutions 24]. We investigate the performance of LoRa in scenarios with mobility, both inside a forest and inside vehicles in an urban area with LoRa technology. To model the behavior and range of communication links, we develop field experiments using hardware prototypes based on LoRa devices. The urban and forest scenarios present different radio propagation conditions, with specific obstacles to electromagnetic waves. Our main performance metrics are the Received Signal Strength Indicator (RSSI), Signal to Noise Ratio (SNR), and Packet Delivery Ratio (PDR).

Radio propagation can be extremely unstable in forests and urban scenarios. Despite the many differences, both environments face similar problems for electromagnetic propagation, especially the multipath phenomenon. In the forest, the main cause is the reflection in the vegetation. In the urban environment, the causes are reflections on buildings and vehicles. The forest represents a hostile environment for radio wave propagation, mainly due to the characteristics of the environment, e.g., vegetation, climate variation, NLoS (Non Line-of-Sight) conditions, among others. Meanwhile, in urban scenarios, 
buildings, speed and changes of elements positioning may generate frequent disconnections. This makes both forest and urban environments complex and challenging for the dissemination of information among wireless network participants.

The use of LoRa technology to collect data in sensor networks has been previously proposed in the literature $[5,6,26,32$. Nevertheless, to the best of our knowledge, this is the first work that analyzes the behavior of LoRa communication links in a tropical forest environment. As a consequence, our challenge in this scenario is to establish the performance of the link with NLoS and speed variations, to guarantee the sending of packets with relevant data like the location of visitors, besides to analyzing the limitations of RSSI implicit in the scenario. It is important to note that the tropical rainforest is characterized by dense vegetation layers that impair transmission quality. We want to assess the feasibility of using LoRa for localisation, of hikers for example. As the accuracy of the localisation relies on the signal quality, we want to observe the signal characteristics in different kind of environments. Thus, we ran experiments not only in tropical rainforest but also in suburban and urban environments. Our urban and suburban communication analysis considers the power received at both ends in a symmetrical half-duplex communication, concerning time parameters between the transmitter and the receiver. Our challenge in this scenario is to analyze the performance of the links, with the presence of few and lots of infrastructure, low and high-density urban areas, and also with a high-speed transmitter mobility.

This paper is organized as follows. In Section 2 , the related works are discussed. Section 3 briefly reviews the main characteristics of LoRa technology. Section 4 describes the setup used in our measurement campaigns in the city and forest environments. The experiment results are presented in Section 5 Section 6 discusses our main observations. Finally, Section 7 concludes the work and points out future challenges.

\section{Related Work}

Several works in the literature confirm the difficulty of electromagnetic propagation trough buildings and vegetation, measuring the signal to characterize its behavior and modeling the power losses.

Rama Rao et al. 29 perform measurements on mango and guava plantations, using the $868 \mathrm{MHz}, 915 \mathrm{MHz}$, and $2.4 \mathrm{GHz}$ bands. Attenuation is measured from the RSSI values and compared with the models proposed by the ITU-R (ITU Radiocommunication Sector) 19 22, Weissberger 33] and COST 235 [16] using Matlab simulations. The authors note that COST 235 was the model closest to the experimental values.

ITU documents also deal with propagation in the frequency bands of interest, as the recommendations P.1546-5 [19], P.1812-4 222], P.1406-2 20] and P.1411-9 21 that present models for terrestrial services. Recommendations P.1546-5 and P.1812-4 propose propagation prediction methods for point-to- 
area terrestrial services, but in a different frequency range, $30 \mathrm{MHz}$ to 3000 $\mathrm{MHz}$ and VHF and UHF bands respectively. P.1406-2 also applies to the VHF and UHF bands but assesses the effect of mobility, whereas recommendation P.1411-9 recommends prediction methods for planning short-range outdoor radio-communication systems in the frequency range $300 \mathrm{MHz}$ to $100 \mathrm{GHz}$. Although these recommendations refer to the same frequency range and pointto-area services, none of them assesses the presence of vegetation. More specifically, the P.833-9 recommendation $\overline{18}$ describes the effects of the signal propagation when one of the radio stations is inside the vegetation. Beyond attenuation also occurs polarization loss, attenuation variation by the wind and temporal spread.

ITU-R reports 236-6 [9, 239-6 [10] and 567-3 [11] also make recommendations on environments with vegetation. However, they have been developed from measurements carried out mainly at UHF. Report 236-6 9 models the vegetation of dense forests and jungles over the level ground as a weak, lossy dielectric slab. The transmitting or receiving antenna are near enough to a small (less than $400 \mathrm{~m}$ deep) grove of trees so that the majority of the signal propagates through the trees. In our study, the frequency used does not allow this slab approximation. Besides, the environment is a tropical forest and not a grove of deciduous trees. In Report 239-6 [10] measurements were made behind deciduous woods in summer and winter, at distances ranging from $10 \mathrm{~km}$ to $200 \mathrm{~km}$, with both transmitter and receiver located above the forest. Again we have a different vegetation environment and a totally different range of distances and location of devices. Report 567-3 11] also discusses vegetation in the link, but in the case where both antennas are outside of a grove of trees, the path can be modeled as a diffraction path. None of these recommendations can be applied to the case treated here, since we consider two stations, transmitting and receiving, within the rainforest vegetation, both providing point-to-area and point-to-point communication services using ISM frequency band.

LoRa has been widely studied by the academic community for different uses and from different perspectives. Several studies $23,26,27,32$ look at the characteristics of the LoRa signal in different settings. Some of them study LoRa under mobility [5, 23, 26, 27]. The general conclusion is that LoRa communication is robust to mobility as long as the devices are moving at a moderate speed. LoRa for geolocation is studied in 12,28 . Results are generally very inaccurate. Accuracy can be improved with computing techniques, but it takes a heavy toll on battery longevity [25]. Specifically, [17] studies the performance of LoRa in continental forest and mountain environments. It is shown that the vegetation and high temperature reduce the range of communication, and that the choice of the antenna greatly impacts the quality of the signal.

The present work differs from the aforementioned literature, as it investigates the propagation both in cities and tropical forests. 


\section{LoRa Background}

Several technologies can be used for sensing in IoT and WSN. The main requirement is low power consumption. Possibilities include Low Power Wide Area Network (LPWAN), Wireless Local Area Network (WLAN), and Wireless Personal Area Network (WPAN), Near-Field Communication (NFC), and cellular IoT communication from the Third Generation Partnership Project (3GPP). In this paper, we focus on LPWAN, as they have been consolidating as an alternative for sensing. LoRa (Long Range) technology, developed by Semtech, is one of the main technological alternatives, especially for applications requiring large area coverage [14]. Although there are other LPWAN technologies, this work focuses on LoRa technology as it is one of the most successful today [30]. We assess the performance of LoRa technology operating on the urban and forest environments using prototype terminal devices, built with off-the-shelf components.

\subsection{LoRa}

LoRa is a technology designed for reduced infrastructure, large coverage and low power consumption. LoRa has the potential to support monitoring and cooperative navigation applications, where small amounts of data need to be transmitted, with no delay guarantees. LoRa is also characterized by reduced hardware complexity, reduced overhead given the small header size, and reduced network complexity in terms of hops and addressing. LoRa operates in the $169 \mathrm{MHz}$ and $430 \mathrm{MHz}$ frequency range in Asia, $433 \mathrm{MHz}$ in Europe, $780 \mathrm{MHz}$ in China, $868 \mathrm{MHz}$ in Europe and $915 \mathrm{MHz}$ in the Americas.

LoRa physical layer (LoRaPHY) was designed to meet the low power requirements of smart objects in smart city applications. LoRaPHY uses Forward Error Correction (FEC) and a proprietary modulation which is a variant of Chirp Spread Spectrum (CSS) 8, 31. LoRaPHY modulates frequency chirp pulses to encode the information by varying the frequency without changing the phase between adjacent symbols [15. The resulting signal is resistant to noise interference or near frequency signals. On top of LoRaPHY, LoRaWAN 24 is the protocol which provides link layer and network layer functionalities. LoRaWAN defines the network topology as a star and Aloha as the Medium Access Control (MAC) protocol. It also defines the dynamic allocation of data rates and provides support for node location services. Unlike LoRaPHY, which is proprietary, the LoRaWAN protocol specification is open and released since 2015. Because the objective of this work is to characterize the propagation only, it was decided not to use LoRaWAN. This choice has the advantage of not restricting tests by the LoRaWAN duty cycle.

Power consumption, transmission range, and interference resistance can be adjusted through four configuration parameters: carrier frequency, which defines the center frequency for the transmission band; bandwidth (BW), which defines the size of the transmission band; coding rate $(\mathrm{CR})$, which defines the 
FEC scheme; and spreading factor (SF), which defines the spectral spreading [8]. The carrier frequency is set according to the region of operation. It is generally not an application choice. Bandwidth, in turn, has three programmable values: $125 \mathrm{kHz}, 250 \mathrm{kHz}$ and $500 \mathrm{kHz}$. SF defines the ratio of bit rate to chirp rate. The specification defines six different values for the spreading factor parameter: SF7, SF8, SF9, SF10, SF11, and SF12 6]. Spectral spreading allow configuration of orthogonal channels so that links with different SF do not collide with each other. Higher SF allows the reception of signals with lower RSSI. It increases the duration of symbols but, as a consequence, decreases the transmission rate 30 . The $\mathrm{CR}$ (coding rate) defines how many bits are used for redundancy in the message to perform error recovery.

\section{Experimental Methodology}

In this work, we study the signal behavior of wireless communications in several different environments: urban (city), sub-urban (country side) and forest environments. Depending on the location and scenario of the experiments, we followed different experimental methodologies. In this section, we detail the specificities of each of those methodologies.

\subsection{Teresópolis headquarters of PARNASO}

PARNASO (Serra dos Órgãos National Park) is a natural conservation unit, with a large part of its area covered by rainforest. Thus, it was chosen to carry out wireless communication tests inside a forest environment. To evaluate the LoRa 14 technology the following metrics are analyzed: radio range, RSSI, SNR, PDR, PIR time, and mobility. SF variation is also evaluated. The shadowing caused by large scale fading and variations caused by small scale fading are studied. Different scenarios were used in the practical experiments designed to analyze separately each type of fading and the impact of mobility. Data is collected to identify the spatial and temporal variation of the signal, especially relevant within the forest. Temporal spreading is not considered due to the use of modulation based on Chirp Spread Spectrum (CSS) by LoRa. The measurement campaign is done at Teresópolis headquarters of PARNASO, specifically at the so-called "suspended trail", an elevated wooden pathway. Two prototype nodes based on Arduino micro-controllers are used to create the measurement scenarios. Figure $1 \mathrm{a}$ shows the test site, and Figures $1 \mathrm{~b}$ and $1 \mathrm{c}$ shows the pole-mounted prototypes and tree-mounted prototypes, respectively. Pole-mounted prototypes are used for measurements where displacement is required. Tree-mounted prototypes are used for longterm measurements. For mobile measurements, the prototypes were carried around instead of mounted in a structure.

We define four experimentation scenarios, as explained next.

$\mathbf{1}^{\text {st }}$ : Short-term static scenario. 


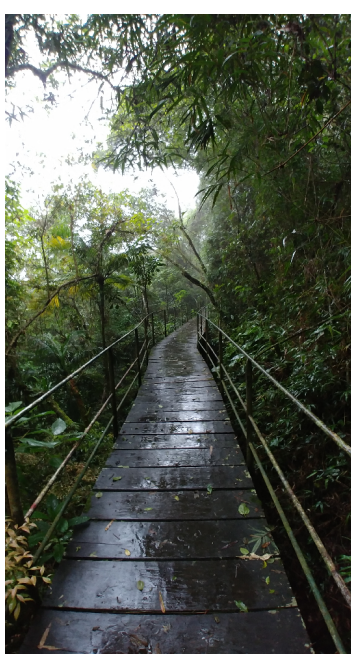

(a) The suspended trail.

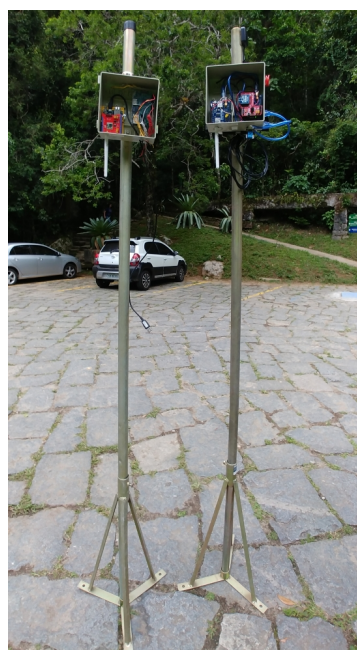

(b) Pole-mounted nodes.

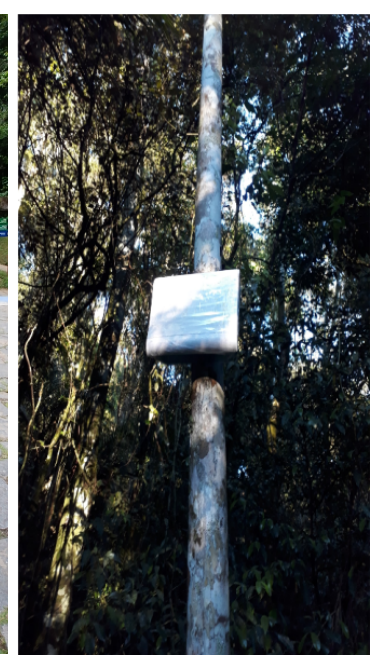

(c) Tree-mounted node.

Fig. 1: Experiment location and prototypes.

The objective of this scenario is to measure the maximum communication range in the forest and trace the behavior of the signal by analyzing its main parameters concerning the distance. Figure 2 illustrates the scenario. Points were selected for the location of the transmitter and for the location of the receiver units to obtain links with length varying by steps of $50 \mathrm{~m}$ until packet reception is no longer possible. Up to $50 \mathrm{~m}$ of distance, the line of sight wasn't totally free of obstacles from the vegetation. For longer distances, vegetation imposes NLoS conditions. For each experiment run, a series of 200 packets are transmitted. Ten runs are performed for each distance.

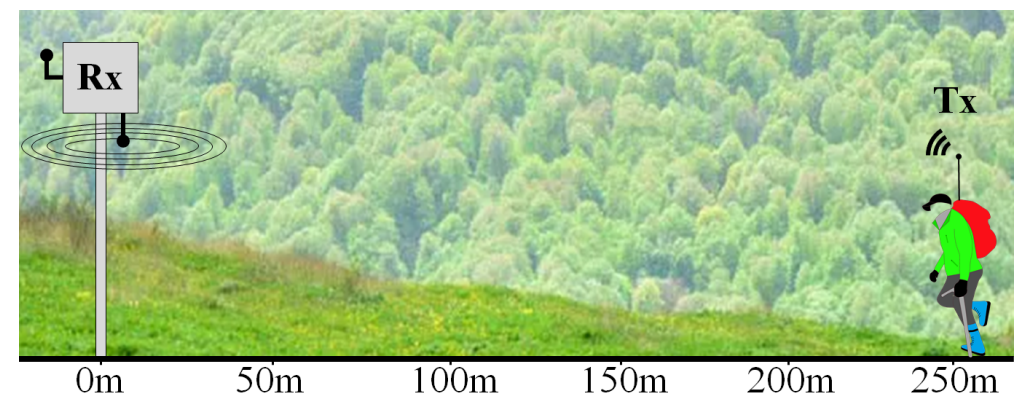

Fig. 2: Short-term static scenario.

$2^{\text {nd }}$ : Long-term static scenario. 
In this scenario we analyze the behavior of the signal over a longer measurement interval. The aim is to identify recurring variations and disruptions. Long-term measurements are performed using LoRa SF12 at a distance of $250 \mathrm{~m}$, during 12 hours. This time range is limited by the batteries that supply the equipment. The line of sight was obstructed by vegetation. The $250 \mathrm{~m}$ distance was chosen for this scenario because it was the maximum range of LoRa in the forest environment, as observed in the short-term static scenario. This $250 \mathrm{~m}$ range is much lower than the theoretical range described in the literature, which refers to open environments under LoS conditions, demonstrating the importance of attenuation by the vegetation.

Long-term measurements indicate that connectivity in the forest environment may behave inconstantly, leading to an intermittent connection and opportunistic communication, when available.

The mobile scenarios introduce the mobility of the nodes, allowing to evaluate the communication between people walking around the forest.

\section{$3^{\text {rd }}$ : One mobile node scenario.}

This scenario is designed to analyze the effect of mobility on the communication link. Figure 3a shows the movement of the transmitter towards the receiver. The transmitter node moves at two different speeds whereas the receiver is fixed. This scenario illustrates a person walking around in the forest, whilst its mobile device communicates with an access point.

\section{$4^{\text {th }}$ : Two mobile nodes scenario.}

This scenario is similar to the previous one, but now both the transmitter and the receiver are mobile. It aims at identifying changes in the signal caused by mobility and intersection of the two nodes. Figure 3b shows the terminals moving toward one another, at the same speed. One starts the movement at the maximum range point $(250 \mathrm{~m})$ and the other starts at the origin point $(0 \mathrm{~m})$, crossing midway and reproducing peer-to-peer communication between two people which cross each other.

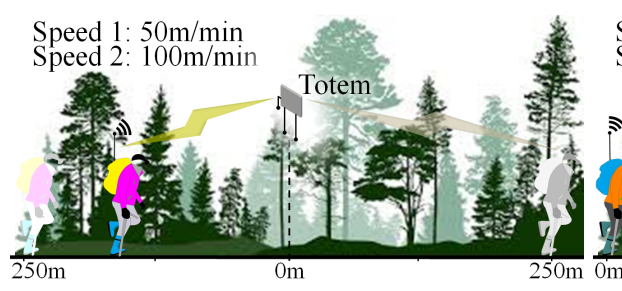

(a) One mobile node scenario.

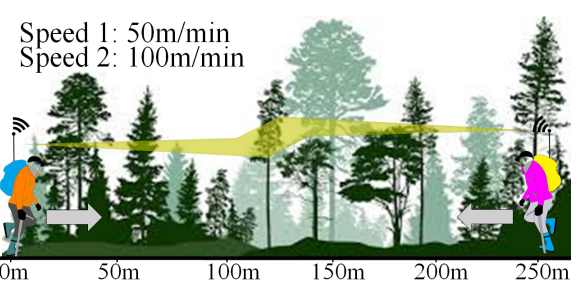

(b) Two mobile nodes scenario.

Fig. 3: Scenarios with mobility

The tests performed in the mobile scenarios use SF12, distance $250 \mathrm{~m}$ as range limit and speeds of $50 \mathrm{~m} / \mathrm{min}(3 \mathrm{~km} / \mathrm{h})$ and $100 \mathrm{~m} / \mathrm{min}(6 \mathrm{~km} / \mathrm{h})$. These speeds are typical of people doing trekking in a forest park. According to the Rio de Janeiro Mountain Sports Federation (FEMERJ), a moderate trail, up 
to $12 \mathrm{~km}$ long and up to $600 \mathrm{~m}$ uphill or $800 \mathrm{~m}$ downhill, can be traveled at an average speed of $6 \mathrm{~km} / \mathrm{h}$ by a well-trained hiker [13]. Two people with this profile, moving at the same speed results in the shortest average contact time between the two terminals. The case of two average people, who move slowly at $3 \mathrm{~km} / \mathrm{h}$, results in a connection lasting longer between their terminals. In both mobile scenarios, the same parameters are evaluated, namely, RSSI, SNR, PDR and contact time. These parameters are related to speed.

As mentioned before, we employ two prototype nodes. The experimental pieces of equipment are summarized in Table 11. We use Arduino UNO controller units and LoRa commercially-available programmable radios. GPS location accuracy is $2.5 \mathrm{~m} 1$.

Table 1: Hardware components used in the LoRa prototype nodes.

\begin{tabular}{|c|c|}
\hline Hardware & Model \\
\hline Controller & Arduino Uno R3 \\
\hline Wireless interface & Dragino RF96 \\
\hline Antenna & Monopole 7dBi \\
\hline GPS Receiver & U-blox NEO-6M \\
\hline Temperature and Humidity Sensor & DHT22 \\
\hline
\end{tabular}

The transmitter unit has a controller that processes location and communication data. Tracking is provided by the GPS receiver. The communication consists of the transmission of data tuples which includes a sequence number, geographic coordinates, timestamp, temperature, and humidity readings. The receiving unit has a controller that processes the data received by the wireless interface. The reception of each packet is logged along with its data, reception timestamp, RSSI, and SNR. RSSI and SNR are computed by the Arduino controller based on measurements done by the wireless interface.

To store the packets transmitted and received, and to obtain timestamps for each tuple of data generated, we run python scripts in two laptops and two Raspberry Pi. For the short-term static, one mobile node, and two mobile nodes scenarios, we use laptops with Ubuntu Linux v.18.04 operating system, 16 GB RAM, an Intel Core i7 processor, and $2 \mathrm{~TB}$ storage. For the long-term static scenario, we use a Raspberry Pi 3 Model B v1.2, with Raspbian Strength operating system, 1 GB RAM, Broadcom 64bit ARM Cortex-A53 Quad-Core Processor and $32 \mathrm{~GB}$ storage. To guarantee the autonomy of the device for at least 12 hours, we use a $10000 \mathrm{mAh}$ power bank.

The configuration parameters of the modules are listed in Table 2 . We have used the $915 \mathrm{MHz}$ frequency band, which is within the frequency plan for restricted radiation equipment in Brazil. We set the transmission power to $14 \mathrm{dBm}$ according to EU868 standard (at the time of our measurement campaign a Brazilian standard was not defined). We have also increased the radiation intensity of the LoRa interface using antennas with a gain of $7 \mathrm{dBi}$, which does not exceed the EIRP (Effective Isotropic Radiated Power) allowed for the AU915-928 frequency plan, established for Brazil. Experiments were 
made for different $\mathrm{SF}$ values $(7,9,12)$. These spreading factors were selected because they describe the behavior of the technology shortly, without the need to test all SF since they are the upper and lower limits plus two intermediate SF. In the short-term static scenario, for each configuration, 10 experiment runs are performed. Each experiment run consists in the transmission of a sequence of 200 packets. For the other three scenarios, packets are sent continuously.

Table 2: Configuration parameters.

\begin{tabular}{|c|c|}
\hline Parameter & Value \\
\hline Frequency & $915 \mathrm{MHz}$ \\
\hline Transmission Power & $14 \mathrm{dBm}$ \\
\hline Spreading Factor & $\mathrm{SF}(7,9,12)$ \\
\hline Bandwidth & $500 \mathrm{kHz}$ \\
\hline Coding Rate & $4 / 5$ \\
\hline
\end{tabular}

\subsection{Lille surroundings}

Here we briefly describe the LoRa radio transceiver which was used, along with the different experimental environments. As our goal is to investigate the feasibility of using LoRa technology for accurate positioning, we wanted to assess the stability of the signal within different environments.

\subsubsection{System setup}

A typical LoRa-based communication network, as defined in the LoRaWAN standard [2] by the LoRa-Alliance, is based on a star-of-stars topology in which gateways relay messages between end-devices and the core network. In this work, we chose to use device-to-device communications, because the network infrastructure has no influence on the device's wireless link characteristics. Thus, we use two devices: a receiver and a transceiver, and we measure their link Received Signal Strength Indication (RSSI) and stability over time in different settings.

We used two B-L072Z-LRWAN1 LoRA ${ }^{\circledR} /$ Sigfox $^{\mathrm{TM}}$ discovery kit devices for our experiments. Those devices contained an onboard Semtech SX1276 transceiver chip. The devices use stubby straight $900 \mathrm{MHz} 50 \mathrm{ohm}$ antennas. We adapted an existing open source firmware, called SX1276 Generic PingPong, to send a customized beacon. The initiator device of the PingPong (Ping sender) is referred to as the transmitter. The device that replies with Pong messages to the initiator is referred to as the receiver.

We used the $868 \mathrm{MHz}$ frequency band and compared different spreading factors. We configured the devices to use a transmission power of $14 \mathrm{dBm}$ (or $25 \mathrm{~mW}$ ), based on our device capabilities and the frequency plan allowed 
Table 3: Experimental settings.

\begin{tabular}{|c|c|}
\hline Parameter & Values \\
\hline Spreading factor & {$[7,8,9,10,11,12]$} \\
\hline Bandwidth & {$[125,250] \mathrm{kHz}$} \\
\hline Coding rate & $4 / 5$ \\
\hline Transmission power & $+14 \mathrm{dBm}$ \\
\hline Carrier frequency & $868.1 \mathrm{MHz}$ \\
\hline Payload size & 32 bytes \\
\hline
\end{tabular}

in France. Table 3 summarizes all the parameters. These settings are chosen according to the European LoRaWAN specification 2.7].

\subsubsection{Considered scenarios}

To characterize the LoRa radio properties with respect to our objectives, we conducted experiments in three kinds of environments. Note that all these scenarios are carried out without line of sight between the two devices.

1. Suburban areas between lab buildings $A \& B$ : as shown in Figure 4a, the transmitter is deployed on the second floor of building A (orange marker on the map). The receiver device is placed in front of building B (white marker on the map). The distance between devices and thus the communication range is approximately $122.5 \mathrm{~m}$. This distance couldn't be longer, because the link between devices was broken if the devices were moved inside the buildings when using a spreading factor of 7 . This is likely to be due to the building's absorption of the wireless signal.

2. Suburban area with mobile transmitter: this scenario takes places in both a suburban zone and a rural zone with a mobile device moving at a speed of approximately $90 \mathrm{~km} / \mathrm{h}$. It is illustrated in Figure $4 \mathrm{~b}$ and Figure $4 \mathrm{c}$ First, on Figure $4 \mathrm{~b}$ we can see the initial setup of the experiment, where the transmitter (white marker on the map) is onboard a vehicle while the receiver is static and on the second floor of building A (orange marker on the map). Note that the receiver is outside of the building. Then on Figure $4 \mathrm{c}$ we can see the trajectory along which the experimental measurements are registered for the mobile device. The vehicle first moves from the white marker to the light green marker. It then moves along the path to the red marker, then from the red marker to the dark green marker. From the dark green marker, it goes back to the light green marker from same path it came. Finally, the last stretch of trajectory followed by the vehicle goes from the light green marker to the blue marker and then back to its initial position (white marker). The environment between the two green markers is mainly rural, while the rest of it is suburban. Notably, the rural environment is kind of a 'bowl' surrounded by embankment. In this scenario, the maximum communication range with correct signal reception 


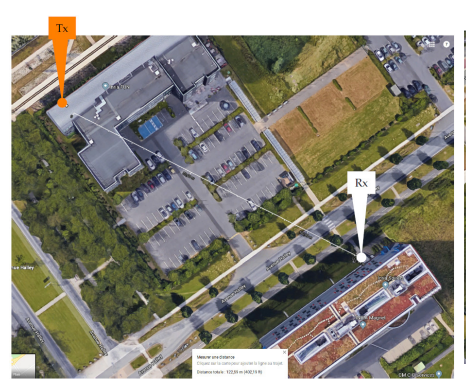

(a) Static suburban scenario.

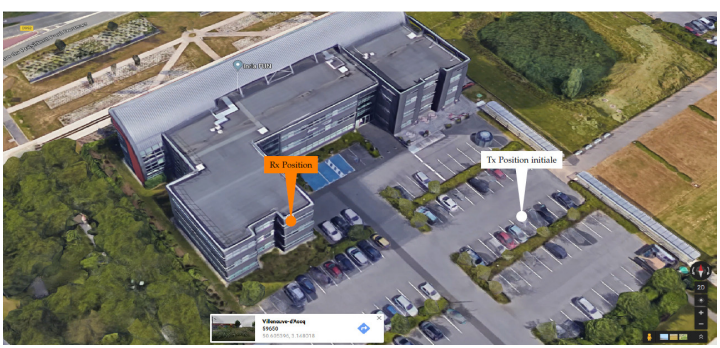

(b) Mobile suburban scenario.

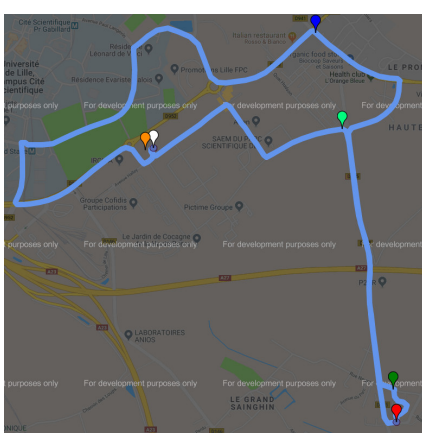

(c) Mobile scenario's trajectory

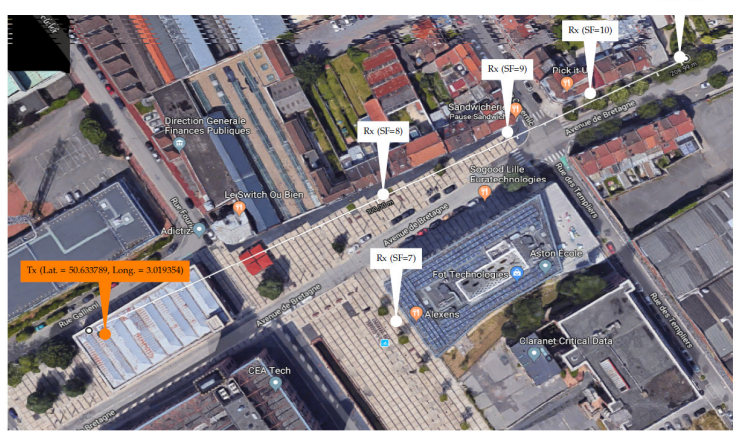

(d) Static urban scenario.

Fig. 4: Urban experiment scenarios at Lille - France.

at both the receiver and transmitter devices is around $1.12 \mathrm{~km}$, while the maximum reached distance is $2.069 \mathrm{~km}$.

3. High density urban area: this experiment was conducted in an environment surrounded by several buildings and with several human activities. We can see the map of this scenario in Figure 4d. Like the first scenario, the transmitter is placed within a room located on the second floor of a building (orange marker on the map), while the receiver is moving around the building to establish the maximum achievable communication distance for each spreading factor. These distances are shown on the map as white markers and reported in Table 4.

On the one hand, the scenarios 1) and 3) are carried out by varying the settings of both the spreading factor and bandwidth as described in Table 3 . For each of those scenarios, the data was measured over half an hour. On the other hand, the mobility scenario is carried out with a fixed spreading factor of value 12 , a fixed bandwidth $B=125 \mathrm{kHz}$, and an experimentation duration equal to the time needed to the drive through the circuit depicted in Figure $4 \mathrm{c}$. 
Table 4: Maximum range per spreading factor.

\begin{tabular}{|c|c|c|c|c|c|c|}
\hline SF & 7 & 8 & 9 & 10 & 11 & 12 \\
\hline Range (m) & 104.22 & 122.91 & 164.98 & 184.49 & 208.30 & 208.96 \\
\hline
\end{tabular}

\section{Signal Behavior}

In this section, we present the results obtained when sending packets between transmitter and receiver from the configurations, described in Section 4 , implemented in the practical experiments of each scenario. The aim is to understand the signal behavior with respect to the specificities of each location and scenario.

\subsection{Teresópolis headquarters of PARNASO}

\subsubsection{Short-term static scenario}

The tests in this scenario mainly aim to evaluate the range of LoRa technology inside the rainforest. PDR, RSSI, and SNR are evaluated as the distance increases. Results are presented with a $95 \%$ confidence interval, represented by vertical error bars in the graphs. As described in Section 4.1, the distance increases by steps of $50 \mathrm{~m}$.

Power measurement is obtained from the RSSI information of the libraries available for the radio modules. Figure 5a shows the RSSI and SNR behavior of the link. Strong attenuation is observed, due to the degradation caused by the vegetation. Looking at the results, we can see that there is an abrupt drop of the metrics in the first $150 \mathrm{~m}$ of the experiment's path, but the signal is still received with a power lower than $-120 \mathrm{dBm}$. This behavior is most noticeable for SF12. Range is significantly reduced in the forest environment, reaching $250 \mathrm{~m}$ with RSSI of $-124 \mathrm{dBm}$. Operation with lower powers is possible due to the spreading of the signal across the spectrum done by CSS modulation, the greater resistance to the temporal spreading of narrow channels, and operation at a relatively low frequency, which suffers less attenuation from the environment.

Figure $5 \mathrm{~b}$ shows the packet delivery ratio of the link, which decreases with distance. As can be seen, SF12 has an anomalous reduction in the PDR, dropping to almost $45 \%$ at $200 \mathrm{~m}$, which may be associated with small-scale fading due to multi-path phenomena and near-point obstacles. The measurement was repeated three times under different atmospheric conditions to try to identify possible causes of this variation. But the severe loss pattern persisted at the $200 \mathrm{~m}$ point. SNR degradation was also observed, as shown in Figure 5a. The low SNR of $-18.73 \mathrm{~dB}$ explains the discrepancy between RSSI and PDR at this point. The PDR may decrease due to errors generated by excessive noise or by small-scale fading. We can explore further this behavior in the long-term testing scenario. 


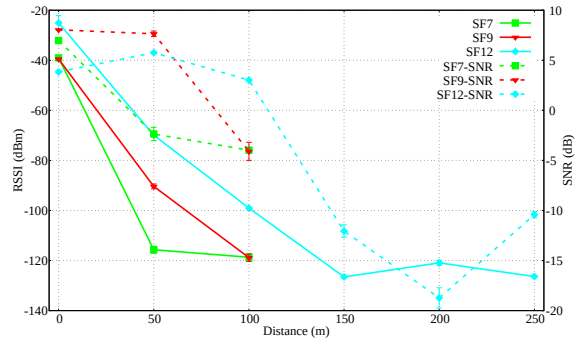

(a) Receiver's RSSI \& SNR for each distance.

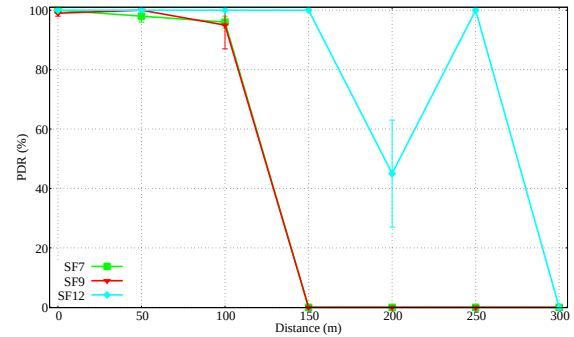

(b) PDR measured for each distance.

Fig. 5: Short-term scenario measurements.

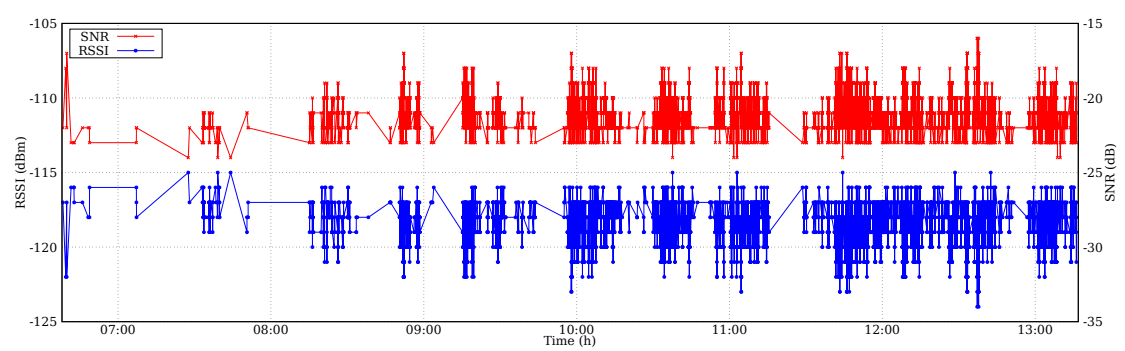

Fig. 6: SF12 long-term test at $250 \mathrm{~m}$.

\subsubsection{Long-term static scenario}

In this scenario, experiments are performed to evaluate the signal variation for a longer time for the characterization of small-scale attenuation. The variation of RSSI, SNR, PDR, and PIR time is observed. Three measurements were recorded, each lasting 12 hours. Terminals are separated by $250 \mathrm{~m}$. This distance corresponds to the maximum radio range in the forest, obtained with LoRa and SF12 in the short-term static scenario. Figure 6 shows part of the measurements and strong signal variations can be noticed. This behavior may be due to the combined multipath and resonance phenomena. Resonance is induced by the absorption of different spectral components by the vegetation, causing peaks and valleys of non-uniform undulation 3 . The multipath is caused by multiple reflections on obstacles, in this case the vegetation, and strongly aggravated by the vegetation moving with the wind.

The PIR and PDR metrics help evaluating the phenomena that occurred. In this measurement, disruptions are clearly identified. The expected PDR in normal operation is greater than $80 \%$, as shown in Figure $5 \mathrm{~b}$. Nonetheless, at many points in time, much lower values are measured, as seen in Figure $7 \mathrm{~b}$ At these points, we observed in Figure $7 \mathrm{a}$ a sharp increase in the PIR time, with an average value well above the latency of $250 \mathrm{~m}$, measured at the fixed scenario as $3 \mathrm{~s}$ in the worst case. The intense variation of PIR at this distance can also be observed through its very large variance, as shown in Table 5. 
These intervals with low PDR produce link disruptions, caused by the random variation of propagation conditions and obstacles.

Table 5: PIR Statistics.

\begin{tabular}{|c|c|}
\hline Measure & Value \\
\hline Measuring point & $250 \mathrm{~m}$ \\
\hline Mean & $3.9205 \mathrm{~s}$ \\
\hline Median & $0.6870 \mathrm{~s}$ \\
\hline Standard deviation & $35.7536 \mathrm{~s}$ \\
\hline Variance & $1278.3192 \mathrm{~s}^{2}$ \\
\hline
\end{tabular}

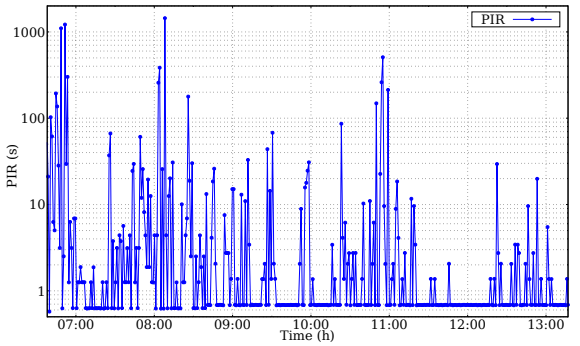

(a) PIR time at $250 \mathrm{~m}$.

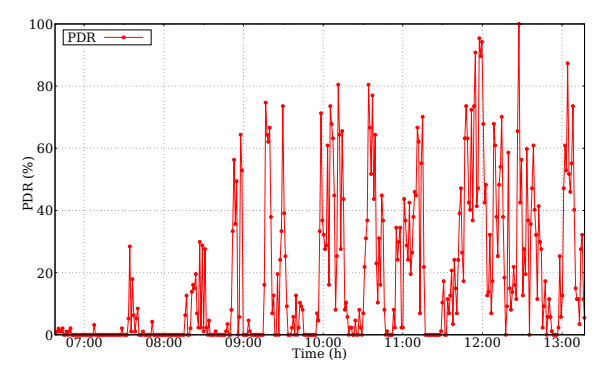

(b) PDR measured for each distance.

Fig. 7: Long-term scenario measurements.

The long-term scenario, together with the short-term one presented in Section 5.1.1, allows us to study the communication between fixed nodes inserted in the vegetation. These scenarios make it possible to assess the range and performance of the link between fixed points, as well as their temporal variability.

\subsubsection{One mobile node scenario}

In this scenario, experiments are performed to simulate the communication between a walker (a visitor to the park) and a fixed base station (the access point). The tests use SF12, SF9 and SF7 with a distance of $250 \mathrm{~m}$ as the range limit. The movement is done on foot, walking at speeds of $50 \mathrm{~m} / \mathrm{min}(3 \mathrm{~km} / \mathrm{h})$ and $100 \mathrm{~m} / \min 6 \mathrm{~km} / \mathrm{h}$ ), repeated ten times for each $\mathrm{SF}$ and speed. These speeds are set based on expected visitor behavior, considering a well-trained practitioner or a slower, average person.

Measurements are executed by moving the transmitter along a path beginning at the maximum range point $(250 \mathrm{~m})$. The terminal continuously transmit packets until it reaches the base station, located at $0 \mathrm{~m}$ mark, and then the terminal returns to the beginning. RSSI, SNR, PDR and Contact Time are 
evaluated for each travel speed. Since $250 \mathrm{~m}$ is the maximum range for SF12, the communication with this SF should cover the whole path. SF7 and SF9 are also evaluated, but their range is smaller, and then coverage is expected to be only partial.

Figures $8 \mathrm{a}$ and $8 \mathrm{~b}$ show the complete displacement continuously, starting at $250 \mathrm{~m}$ passing through the fixed radio at $0 \mathrm{~m}$ and returning to $250 \mathrm{~m}$, as a continuous sequence to ease the visualization of the signal behavior. This movement is represented on the y-axis, on the left scale, as a one-way walk, indicated by the arrow. The scale on the right shows the elapsed time. For the experiment at $50 \mathrm{~m} / \mathrm{min}$, shown in Figure $8 \mathrm{a}$, this time is 10 minutes and for the experiment at $100 \mathrm{~m} / \mathrm{min}$ (Figure 8b), it is 5 minutes.

The variation of RSSI and SNR is represented by the color variation of the various samples plotted in the graphs. Table 6 reports the number of samples received for each speed and SF. In both figures the ranges for RSSI and SNR are identical, indicating the same maximum and minimum values. Nonetheless, the larger speed produces a small reduction in the level of RSSI and SNR, which can be seen through the lighter colors in Figure $8 \mathrm{~b}$.

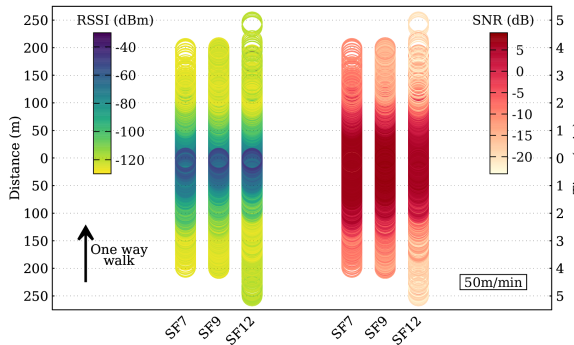

(a) RSSI and SNR at $50 \mathrm{~m} / \mathrm{min}$.

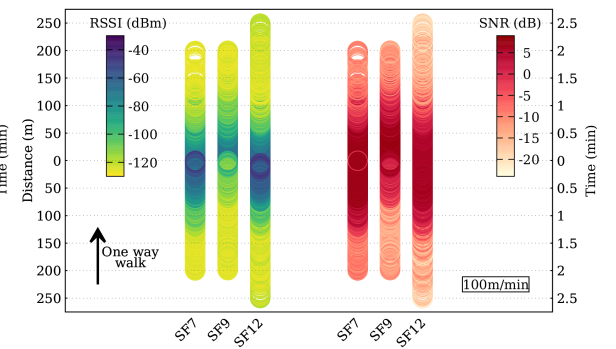

(b) RSSI and SNR at $100 \mathrm{~m} / \mathrm{min}$.

Fig. 8: One mobile node scenario measurements.

During slow travel, terminals send and receive packets over a longer interval. There is, therefore, a larger number of samples with low RSSI and low SNR when the terminals are at the extreme point, and, likewise, a larger number of samples with high values when the terminal is close to the base station. This can be observed by the darkest colors in Figure 8a. The power values remain consistent with those measured in the fixed point scenario, ranging from $-120 \mathrm{dBm}$ at maximum distance to $-40 \mathrm{dBm}$ at the $0 \mathrm{~m}$ mark for RSSI and $-20 \mathrm{~dB}$ to $5 \mathrm{~dB}$, for SNR. There is a slight variation with the SF, allowing the reception of weaker signals using SF12.

Figures $9 \mathrm{a}$ and $9 \mathrm{~b}$ show the variation of the PDR with the speeds of $50 \mathrm{~m} / \mathrm{min}$ and $100 \mathrm{~m} / \mathrm{min}$, respectively. The incongruity found at the $200 \mathrm{~m}$ point of the fixed scenario described in Section 5.1.1 does not repeat here. Nevertheless, PDR values are lower, due to the mobility. The increase in the 
Table 6: Number of samples for displacement at $50 \mathrm{~m} / \mathrm{min}$ and $100 \mathrm{~m} / \mathrm{min}$.

\begin{tabular}{|c|c|c|}
\hline Spreading Factor & Samples Rx at $\mathbf{5 0 ~} \mathbf{~ m} / \mathbf{m i n}$ & Samples Rx at 100 m/min \\
\hline SF7 & 8,883 & 4,856 \\
\hline SF9 & 10,624 & 5,228 \\
\hline SF12 & 5,522 & 3,030 \\
\hline
\end{tabular}

randomness of the received signal is probably due to the vegetation's movement in addition to the terminal's mobility.

At the extreme of the curves, the higher speed seems to bring a greater $\mathrm{PDR}$, but after $200 \mathrm{~m}$ the slope of the curve to $50 \mathrm{~m} / \mathrm{min}$ is greater, indicating a faster growth of the PDR. The graphs are asymmetric, showing differences in the PDR during the approximation and separation of the fixed base station. Remembering that the execution times of the experiments are different for each speed, the measurements made for the speed of $50 \mathrm{~m} / \mathrm{min}$, shown in Figure $9 \mathrm{a}$ took 10 minutes and the measurements for $100 \mathrm{~m} / \mathrm{min}$ took 5 minutes, in Figure $9 \mathrm{~b}$. This is represented on the x-axis, on the upper scale. The bottom scale shows the distance traveled as a continuous path in the same way as in Figures $8 \mathrm{a}$ and $8 \mathrm{~b}$

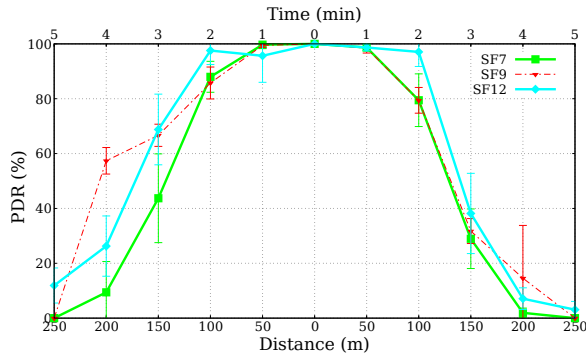

(a) PDR at $50 \mathrm{~m} / \mathrm{min}$

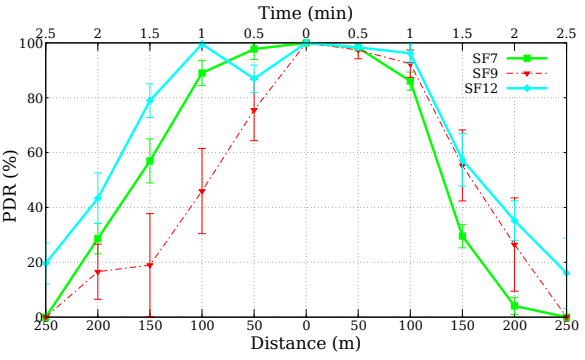

(b) PDR at $100 \mathrm{~m} / \mathrm{min}$.

Fig. 9: One mobile node scenario measurements.

\subsubsection{Two mobile nodes scenario}

This scenario simulates the communication of two people crossing while they follow a trail in the forest. Similar to Section 5.1.3. RSSI, SNR, PDR and contact time are evaluated for two travel speeds. The distance of $250 \mathrm{~m}$ is used again, since it is the maximum range for LoRa in the forest environment. For $\mathrm{SF} 12$, it is expected that there is communication through all the path, which should not be the case for SF7 and SF9. The experiments were repeated ten times for each SF and speed.

Figures 10, 11a, 11b 12a, and 12b show the behavior of the signal according to the terminal speed and distance. The increase in speed brought spacing and 
a reduction in the number of samples received, as can be seen in Figure 10 and Table 7
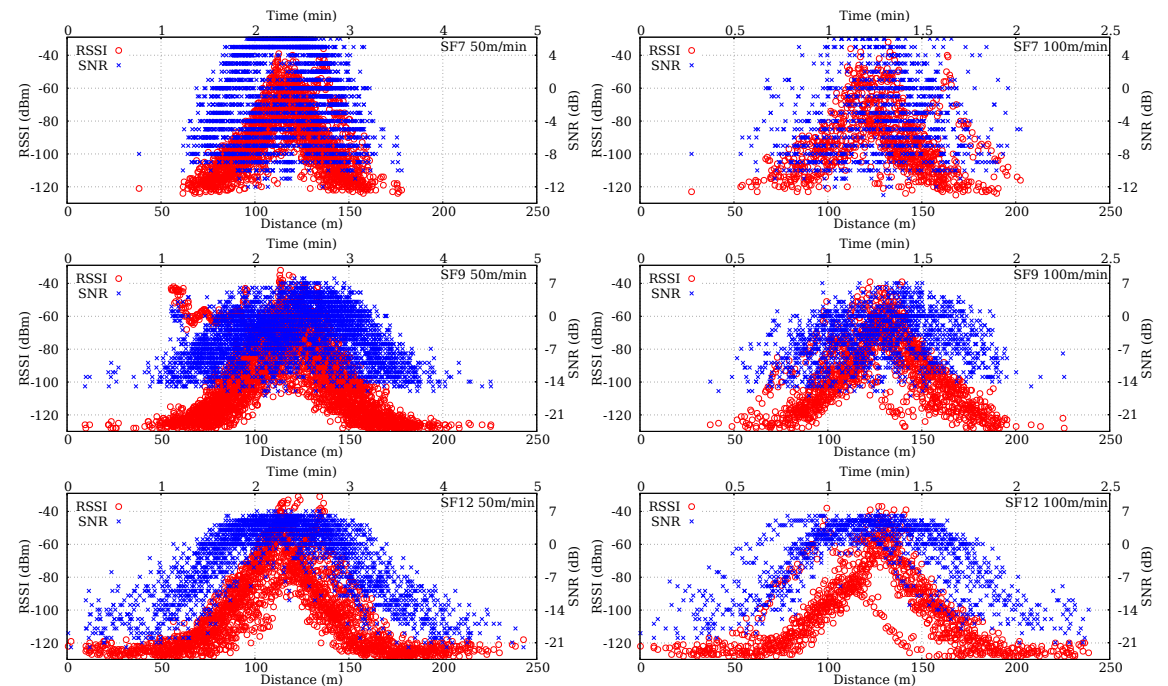

Fig. 10: RSSI and SNR at $50 \mathrm{~m} / \mathrm{min}$ and $100 \mathrm{~m} / \mathrm{min}$.

Table 7: Number of samples for displacement at $50 \mathrm{~m} / \mathrm{min}$ and $100 \mathrm{~m} / \mathrm{min}$.

\begin{tabular}{|c|c|c|}
\hline Spreading Factor & Samples Rx at $\mathbf{5 0 ~} \mathbf{~ m}$ / min & Samples Rx at $\mathbf{1 0 0 ~} \mathbf{~} / \mathbf{m i n}$ \\
\hline SF7 & 2,419 & 1,006 \\
\hline SF9 & 3,817 & 1,609 \\
\hline SF12 & 2,310 & 1,136 \\
\hline
\end{tabular}

Nevertheless, the speed variation does not produce many variations in the RSSI or SNR levels. We can see that Figures 11a and 11b have the same extreme limits for RSSI and SNR,regarding however, the variation in this range differs. In general, there is a slight reduction in the quality of the received signal as the speed increases. In both Figures 11a and 11b the y-axis, on the left scale, reports the distance from the receiving terminal to the origin, that is, its position, instead of the relative distance between the two terminals. Thus, the crossing point, where the power is strongest, occurs in the middle of the band. The y-axis, on the right scale, also reports the time elapsed during the measurement. In Figure 11a the total time is 5 minutes and in Figure 11b, it is 2.5 minutes.

Similar to Section 5.1 .3 when travelling at $50 \mathrm{~m} / \mathrm{min}$, terminals send and receive more packets, so the measurements better show the variation in the sampled values. For the current scenario, this can be observed by the gradual 


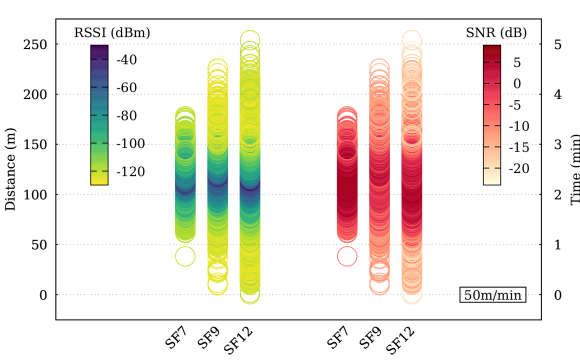

(a) RSSI and SNR at $50 \mathrm{~m} / \mathrm{min}$.

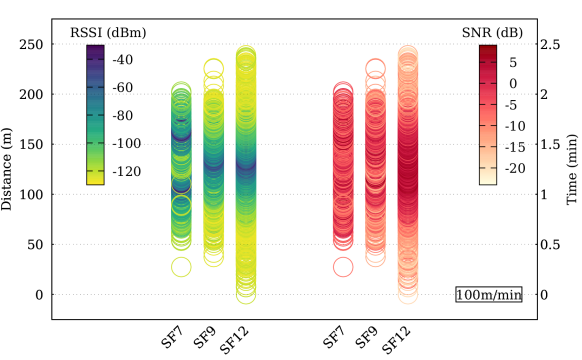

(b) RSSI and SNR at $100 \mathrm{~m} / \mathrm{min}$.

Fig. 11: Two mobile nodes scenario measurements.

variation of colors in Figure 11a, concentrating the darkest tones in the center of the band and the lightest at the tips. Figure $11 \mathrm{~b}$ shows colors that mix more, indicating that the values of the samples vary faster. The range of RSSI and SNR are the same as in the one mobile node scenario.

The variation of the PDR with the speed is shown in Figures $12 \mathrm{a}$ and $12 \mathrm{~b}$ An increase in speed causes a reduction in the PDR and its growth rate, more noticeable for SF7 and SF9. Except for SF7, the PDR for advancing and departing from the terminals is similar for $100 \mathrm{~m} / \mathrm{min}$. In this figure, just like in the previous ones, we should note that the time taken to obtain the measurements is not the same. The measurements made for the speed of $50 \mathrm{~m} / \mathrm{min}$ took 5 minutes and the measurements for $100 \mathrm{~m} / \mathrm{min}$ took 2.5 minutes. Nevertheless, it is possible to observe the interval during which there is packet reception, that is, contact time and the quality of communication during this period.

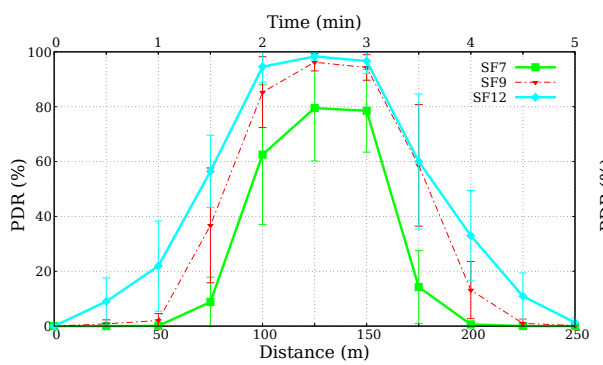

(a) PDR at $50 \mathrm{~m} / \mathrm{min}$.

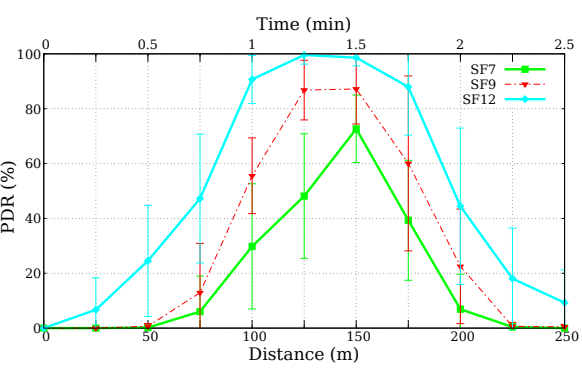

(b) PDR at $100 \mathrm{~m} / \mathrm{min}$.

Fig. 12: Two mobile nodes scenario measurements.

Table 8 describes the average contact time measured for each speed and SF. Similar to Section 5.1.3 the lower the spreading factor, the shorter the range and therefore the contact time. 
Table 8: Contact time in minutes.

\begin{tabular}{|c|c|c|}
\hline Spreading Factor & $\mathbf{5 0} \mathbf{~ m} / \mathbf{m i n}$ & $\mathbf{1 0 0} \mathbf{~ m} / \mathbf{m i n}$ \\
\hline SF7 & $1.81 \pm 0.25$ & $0.87 \pm 0.11$ \\
\hline SF9 & $2.97 \pm 0.50$ & $1.27 \pm 0.25$ \\
\hline SF12 & $3.94 \pm 0.25$ & $2.07 \pm 0.40$ \\
\hline
\end{tabular}

\subsection{Lille surroundings}

This subsection briefly describes the obtained results, based on the different experimental environments and scenarios around Lille city. We consider the three different scenarios regarding the Section 4.2 .2 description. For the scenarios suburban area between Lab building $A \& B$ and high density urban area, we measured the RSSI, SNR and the Packet Delivery Ratio (PDR) of the signal. While the Suburban area with mobile transmitter scenario, due to the loss of log stream from the LoRa radio receiver board, only exhibits the radio signal strength variation over time. Therefore the PDR, which is the ratio between the number of received and transmitted packets for both sides, can not be correctly computed.

\subsubsection{Suburban areas between Lab building $A \& B$}

Figure 13 and Figure 14 respectively describe the radio signal strength variation over time and the PDR for each couple of bandwidth and spreading factor. Figure 13 is a small sample of the results, the full results can be found in the appendix A Each sub-figure presents the signal strength (i.e., RSSI) and the signal noise ratio (i.e., SNR) over time respectively. The top sub-figure represents the RSSI while the bottom one represents the SNR. According to the Figure 13 in this scenario, independently of the spreading factor (SF) and bandwidth (BW), the RSSI and the signal noise ratio, shows an almost similar behavior with a similar average value for both devices. The results of the transceiver and receiver is very similar. However the RSSI is not stable with time for both devices, most likely because of the dynamic environment.

Figure 14 describes the PDR, the erroneous packet due to CRC Error and the packet loss ratio, via a stacked bar chart, for each group of receiver $(\mathrm{Rx})$ and transmitter $(\mathrm{Tx})$ devices respectively, and for each SF. While Figure 14a these performance metrics for LoRa radio with bandwidth equal to $125 \mathrm{khz}$, the Figure 14b exhibits these performance metrics for bandwidth equal to 250 khz. As shown in Figure 14, regardless the couple of parameters (SF and BW), we observe a PDR significantly higher that $95 \%$ while these two other metrics, error rate and loss rate share the remainder ratio, which is less than $4 \%$. Therefore, we can see that in this static scenario, the small variations in the signal metrics does not impact the PDR of the devices. 

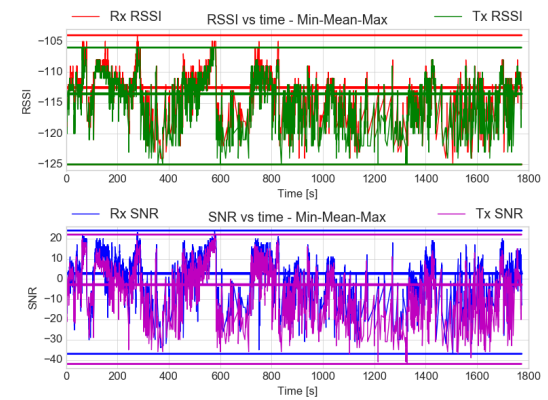

(a) RSSI \& SNR $(\mathrm{SF}=7 \mathrm{BW}=125 \mathrm{kHz})$.

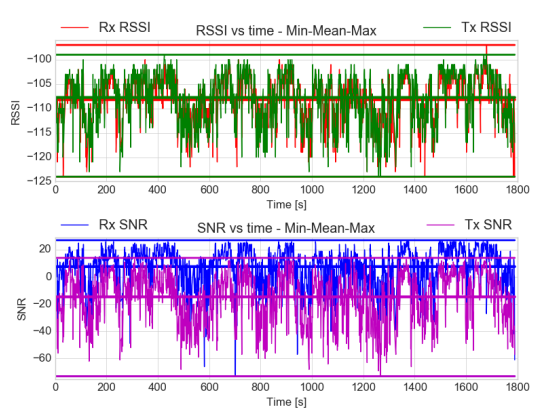

(b) RSSI \& SNR ( $\mathrm{SF}=11 \mathrm{BW}=250 \mathrm{kHz})$.

Fig. 13: RSSI \& SNR values over time for Transmitter and Receiver.

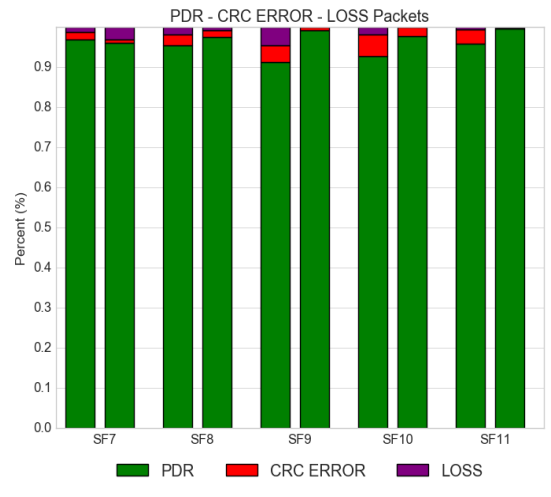

(a) Bandwidth $=125 \mathrm{kHz}$.

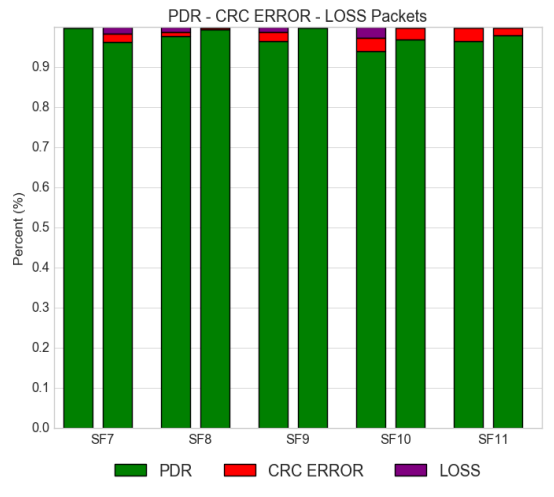

(b) Bandwidth $=250 \mathrm{kHz}$.

Fig. 14: PDR, CRC error packets and Packet Loss Ratio.

\subsubsection{Suburban area with mobile transmitter}

Figure 15 shows the results obtained from experiments in scenario (2). As seen in Figure $4 \mathrm{c}$, the distance from the lab increase with time as we drive away and then decrease as we come back. Thus, we can deduce that as the distance increases (respectively decreases), the RSSI value decreases (respectively increases). Both devices exhibit an almost similar behavior with their closest average value when they are able to receive each other beacon.

According to these results, we can observe three phases. The first phase corresponds to the moment when there is a quasi-symmetric communication between the receiver and the transmitter. During this moment, both signals 
are quite similar and it corresponds to the first $1.12 \mathrm{~km}$ as explained in Section 4.2.2. It lasts from the white marker visible on Figure 4c to the light green marker. The second phase corresponds to the rural environment shaped like a bowl and it lasts from the light green marker to the dark green marker while passing through the red marker. During the vehicle traveling time, there is almost no communication between the transmitter and the receiver in both directions. The exception was at the place marked with the green marker, where few receptions were recorded, most likely because that place was higher in altitude than the rest of the surroundings. The third phase corresponds to the moment when the vehicle is the closest to the light green marker. During this phase, we observe a symmetrical communication between the two devices. In the end of the experiment run, we come back to the lab, and we can see that as the transceiver becomes closer to the receiver, the RSSI value increases. Around time step $1000 \mathrm{~s}$ on Figure 15, we see that the computer logging data from the receiver encountered a system failure, but it did not affect the receiver's operations.
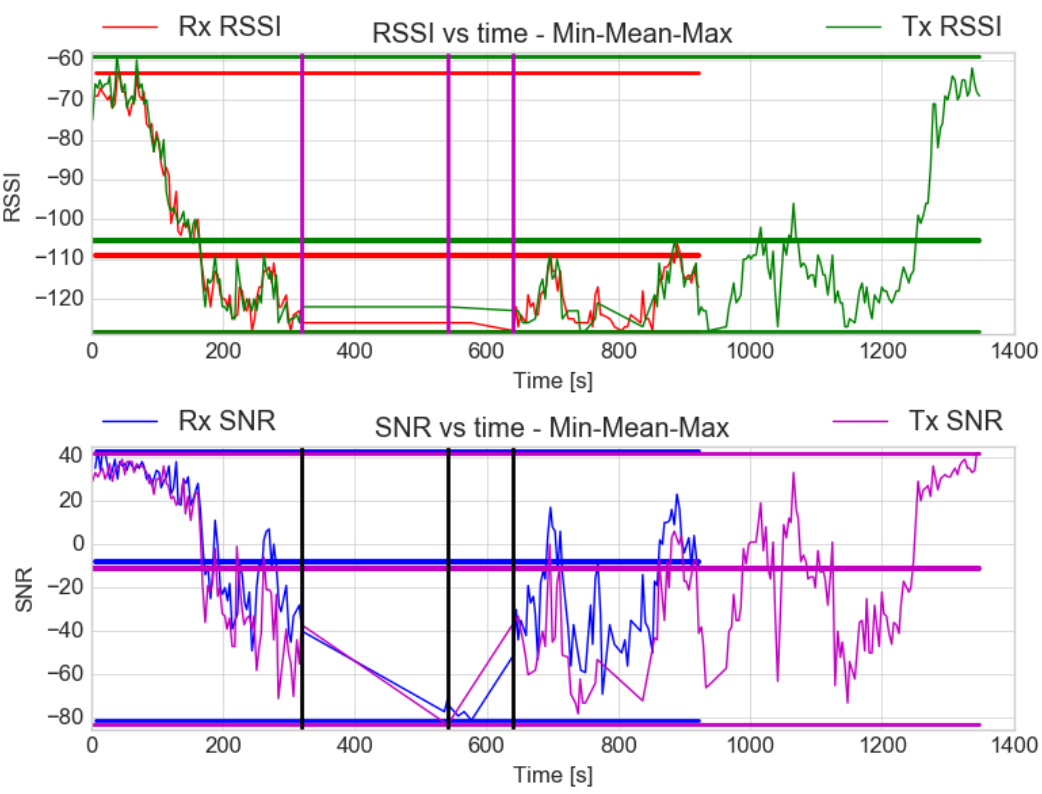

Fig. 15: RSSI \& SNR values over time for $\mathrm{SF}=12$ and $\mathrm{BW}=125 \mathrm{kHz}$ (Mobile).

\subsubsection{High density urban area}

Figure 16 depicts a sample of the results measured in a dense urban environment for different spreading factor and bandwidth values. The full results can be found in appendix B. In this study, we focus our analysis on the most im- 
portant aspects observed for one couple of $\mathrm{SF}=9$ and $\mathrm{BW}=125 \mathrm{khz}$ values for the signal strength variation over time for both devices. Unlike the results from the previous scenarios, here we can see that the signal characteristics from both devices are very asymmetric. This behavior can also be observed for different spreading factor and bandwidth values in [4]. The greater the bandwidth, the more asymmetric are the signals. This is due to the fact that a bigger bandwidth allows a higher transmission rate, which makes the signal more sensitive to errors. This effect is even stronger on the transmitter as the metallic structure of building, weather, and human activities significantly impact the signal propagation.

Figure 17 shows the PDR, the erroneous packet due to CRC Error and the packet loss ratio, via a stacked bar chart, for transmitter (Tx) and receiver $(\mathrm{Rx})$ devices respectively, and for each spreading factor (SF). Figure 17a and Figure $17 \mathrm{~b}$ respectively show these performance metrics for bandwidth equal to $125 \mathrm{khz}$ and $250 \mathrm{khz}$. For a bandwidth of $125 \mathrm{khz}$, except the SF values 9 and 10, specifically for the $\mathrm{SF}=9$, the $\mathrm{PDR}$ is greater than or equal to $80 \%$ for Tx. While it is more than $90 \%$ for $\mathrm{Rx}$. This is explained by the same conclusions previously made for the signal behavior. Unfortunately, the same observations made for the characteristics of the signal can be made for a 250 $\mathrm{kHz}$ bandwidth for the spreading factor 8, 9, 10 and 12 . While the first three spreading factors have a reception rate below $80 \%$ regardless of the identity of the device (i.e. Tx or $\mathrm{Rx}$ ), the last spreading factor presents a reception rate below $60 \%$ only with the transmitter. Note that, regardless of the couple of SF and $\mathrm{BW}$, the erroneous packet ratio is always lower than the packet loss ratio. Moreover, these observations are highly correlated with the signal stability. Therefore, we can draw the same conclusion as in Section 5.2.1. More stable and similar are the signal on both sides, more important is the packet delivery ratio (PDR) of each device.
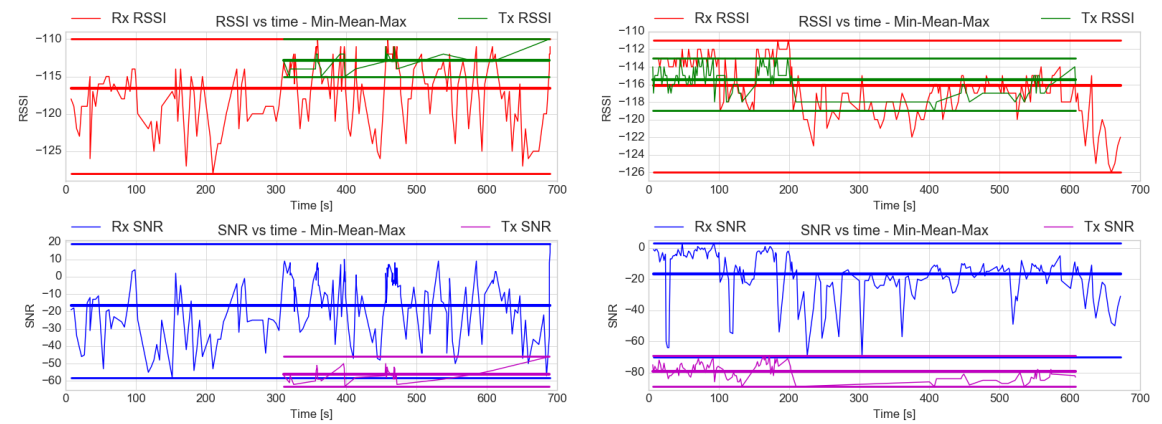

(a) RSSI \& SNR $(\mathrm{SF}=9 \mathrm{BW}=125 \mathrm{kHz})$.

(b) RSSI \& SNR $(\mathrm{SF}=12 \mathrm{BW}=250 \mathrm{kHz})$.

Fig. 16: RSSI \& SNR values over time for Transmitter and Receiver. 


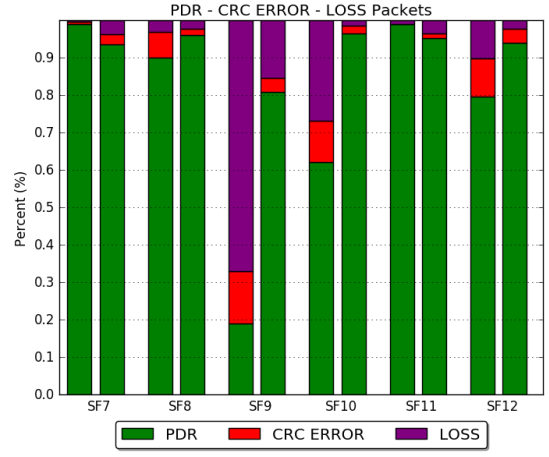

(a) PDR - CRC ERROR - Pack. LOSS.

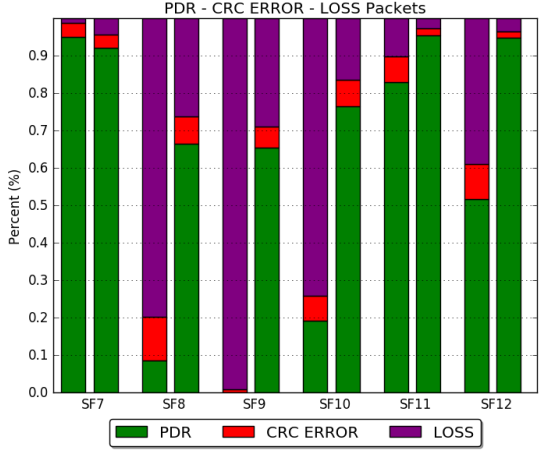

(b) PDR - CRC ERROR - Pack. LOSS.

Fig. 17: Packet Delivery Ratio (PDR), CRC error packets and Packet Loss Ratio.

\section{Discussion}

In this study, we tested experimentally LoRa communications in various environments. From the obtained results, we can deduce several things. First, from the experiments conducted near Lille, we know that using two LoRa devices in a device-to-device fashion instead of the classical device-to-basestation greatly reduces the communication range. The maximum communication range reached is much shorter than the previously cited studies that followed a standard device-to-gateway communication paradigm. Also, in compliance with [26], LoRa devices configured with a spreading factor of 12 and moving at a moderate speed $(\simeq 40 \mathrm{~km} / \mathrm{h})$ do not disrupt LoRa communications. While traveling at a high-speed, around $\simeq 90 \mathrm{~km} / \mathrm{h}$, greatly increases link breaking probability. Besides, LoRa signal stability is greatly depending on the environment. In a rural environment, the signal appears to be more stable than in an urban zone. This is mainly due to the presence of less obstacles in the area, which means less signal reflection.

This in turn is confirmed by the experiments conducted in PARNASO. LoRa was designed for long-range, with theoretical coverage of $15 \mathrm{~km}$ for suburban and $5 \mathrm{~km}$ for urban areas. Nevertheless, measurements in the forest had an extremely short range compared to the theory. The maximum measured range is $250 \mathrm{~m}$ for SF12. This drastic reduction is due to the difficult propagation conditions in the forest environment. LoRa has been shown to be sensitive to the presence of obstacles and reflectors despite the CSS modulation technique that is more robust against interference. The behavior of RSSI and PDR is similar in both mobile scenarios. The main effect perceived by the small variation in speeds was only a reduction in the contact time and, consequently, in the number of packets received. 


\section{Conclusion}

In this paper we presented a study of the electromagnetic propagation under various environments, cities as well as rainforests. Our aim is to understand how could LoRa be used for alternative applications such as geolocation, of hikers in a natural park for example. According to these results and analysis, we can conclude that the LoRa signal stability greatly depends on the environment and it is more stable in suburban areas than in high density urban areas. As future work, we wish to further study the impact of the environment, in terms of atmospheric conditions (air humidity, pressure, etc.), on the LoRa performance, and propose a suitable propagation model for rainforests from the obtained results.

\section{References}

1. AG, U.H.: NEO-6: U-blox 6 GPS modules data sheet (2011). URL https: //www.u-blox.com/sites/default/files/products/documents/NEO-6_DataSheet_ (GPS.G6-HW-09005).pdf Accessed on Dec. 2019

2. Alliance, L.: LoRaWAN specification. https://lora-alliance.org/resource-hub/ lorawanr-specification-v103 (2015)

3. Alves, G.C.G.: Modelagem da propagação radiomóvel no ambiente cerrado nas faixas denominadas VHF/UHF. Ph.D. thesis, Universidade de Brasília (2015). (In portuguese)

4. Amadou, I.: https://github.com/iamadou/LoRa-Testbed-Measures (2019)

5. Andrei, M.L., Rădoi, L.A., Tudose, D.S.: Measurement of node mobility for the LoRa protocol. In: IEEE RoEduNet 2017. Targu Mures, Romania (2017)

6. Augustin, A., Yi, J., Clausen, T., Townsley, W.M.: A study of LoRa: Long range \& low power networks for the Internet of things. Sensors 16(9), 1466 (2016)

7. Bargen, D.: LoRaWAN data rates. https://blog.dbrgn.ch/2017/6/23/ lorawan-data-rates/ (2017)

8. Bor, M., Vidler, J., Roedig, U.: LoRa for the internet of things. In: International Conference on Embedded Wireless Systems and Networks (EWSN), pp. 361-366 (2016)

9. Dubrovnik, C.X.P.A.: Volume v: Propagation in non-ionized media. report 236-6 influence of terrain irregularities and vegetation tropospheric propagation. Tech. rep., International Telecommunication Union, Geneva (1986)

10. Dubrovnik, C.X.P.A.: Volume v: Propagation in non-ionized media. report 239-6 propagation statistics required for broadcasting services using the frequency range 30 to 1000 mhz. Tech. rep., International Telecommunication Union, Geneva (1986)

11. Dubrovnik, C.X.P.A.: Volume v: Propagation in non-ionized media. report 567-3 methods and statistics for estimating field-strength values in the land mobile services using the frquency range $30 \mathrm{mhz}$ to $1 \mathrm{ghz}$. Tech. rep., International Telecommunication Union, Geneva (1986)

12. Fargas, B.C., Petersen, M.N.: GPS-free geolocation using LoRa in low-power WANs. In: IEEE GIoTS 2017. Geneva, Switzerland (2017)

13. FEMERJ: Metodologia de classificação de trilhas. In: Report FEMERJ: No STE2015/01, Federação de Esportes de Montanha do Estado do Rio de Janeiro - FEMERJ (2015). (In portuguese)

14. Foubert, B., Mitton, N.: Long-Range wireless radio technologies: A survey. Future Internet 12(13) (2020)

15. Goursaud, C., Gorce, J.M.: Dedicated networks for IoT : PHY / MAC state of the art and challenges. EAI endorsed transactions on Internet of Things (2015)

16. Hall, M.: COST project 235 activities on radiowave propagation effects on nextgeneration fixed-service terrestrial telecommunication systems. In: 8th International Conference on Antennas and Propagation, pp. 655-659. IET (1993) 
17. Iova, O., Murphy, A.L., Picco, G.P., Ghiro, L., Molteni, D., Ossi, F., Cagnacci, F.: Lora from the city to the mountains: Exploration of hardware and environmental factors. In: Proceedings of the 2017 International Conference on Embedded Wireless Systems and Networks (2017)

18. ITU-R: P.833-9 attenuation in vegetation. Tech. rep., International Telecommunication Union, Geneva (2012)

19. ITU-R: P.1546-5 method for point-to-area predictions for terrestrial services in the frequency range $30 \mathrm{mhz}$ to $3000 \mathrm{mhz}$. Tech. rep., International Telecommunication Union, Geneva (2013)

20. ITU-R: P.1406-2 propagation effects relating to terrestrial land mobile and broadcasting services in the vhf and uhf bands. Tech. rep., International Telecommunication Union, Geneva (2015)

21. ITU-R: P.1411-9 propagation data and prediction methods for the planning of shortrange outdoor radiocommunication systems and radio local area networks in the frequency range $300 \mathrm{mhz}$ to $100 \mathrm{ghz}$. Tech. rep., International Telecommunication Union, Geneva (2015)

22. ITU-R: P.1812-4 a path-specific propagation prediction method for point-to-area terrestrial services in the vhf and uhf bands. Tech. rep., International Telecommunication Union, Geneva (2015)

23. Liando, J.C., Gamage, A., Tengourtius, A.W., Li, M.: Known and unknown facts of LoRa: Experiences from a large-scale measurement study. ACM Trans. Sen. Netw. 15(2), 16:1-16:35 (2019)

24. LoRa ${ }^{\mathrm{TM}}$-Alliance: LoRa ${ }^{\mathrm{TM}}$-alliance technology. Available at: https://www . lora-alliance.org/technology (2016). Accessed February 2019

25. Mackey, A., Spachos, S.: LoRa-based localization system for emergency services in GPSless environments. In: IEEE Workshop WCNEE 2019. Paris, France (2019)

26. Petajajarvi, J., Mikhaylov, K., Pettissalo, M., Janhunen, J., Iinatti, J.: Performance of a low-power wide-area network based on LoRa technology: Doppler robustness, scalability, and coverage. International Journal of Distributed Sensor Networks 13 (2017)

27. Petajajarvi, J., Mikhaylov, K., Roivainen, A., Hanninen, T., Pettissalo, M.: On the coverage of LPWANs: range evaluation and channel attenuation model for LoRa technology. In: IEEE ITST 2015. Copenhagen, Denmark (2015)

28. Podevijn, N., Plets, D., Trogh, J., Martens, L., Suanet, P., Hendrikse, K., Joseph, W.: TDoA-based outdoor positioning with tracking algorithm in a public LoRa network. Wireless Communications and Mobile Computing (2018)

29. Rao, T.R., Balachander, D., Kiran, A.N., Oscar, S.: RF propagation measurements in forest \& plantation environments for wireless sensor networks. In: International Conference on Recent Trends in Information Technology, pp. 308-313 (2012)

30. Raza, U., Kulkarni, P., Sooriyabandara, M.: Low power wide area networks: An overview. IEEE Communications Surveys Tutorials 19(2), 855-873 (2017)

31. Reynders, B., Pollin, S.: Chirp spread spectrum as a modulation technique for long range communication. In: Symposium on Communications and Vehicular Technologies (SCVT), pp. 1-5. IEEE (2016)

32. Wang, S., Chen, Y., Chen, T., Chang, C., Cheng, Y., Hsu, C., Lin, Y.: Performance of LoRa-based IoT applications on campus. In: IEEE VTC-Fall 2017. Toronto, Canada (2017)

33. Weissberger, M.A.: An initial critical summary of models for predicting the attenuation of radio waves by trees. Final Report Electromagnetic Compatibility Analysis Center (1982) 


\section{Appendices}

A Suburban environment between Lab building A \& B
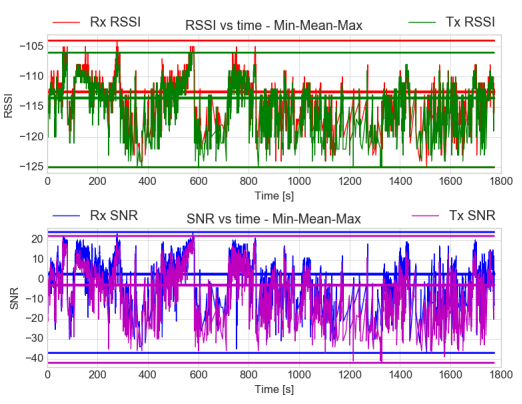

(a) RSSI \& SNR $(\mathrm{SF}=7 \mathrm{BW}=125)$
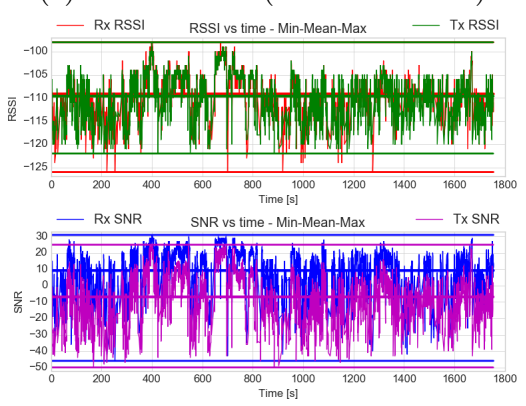

(c) RSSI \& SNR $(\mathrm{SF}=8 \mathrm{BW}=125)$
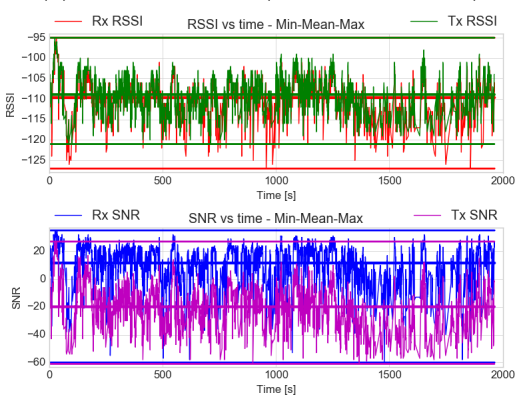

(e) RSSI \& SNR $(\mathrm{SF}=9 \mathrm{BW}=125)$
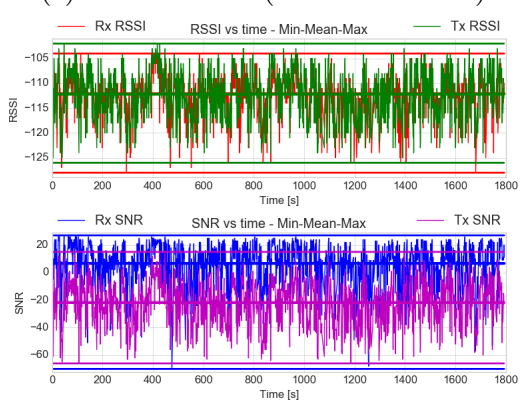

(g) RSSI \& SNR (SF=10 BW=125)

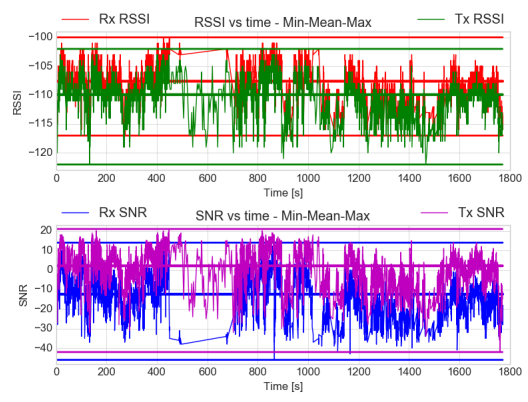

(b) RSSI \& SNR $(\mathrm{SF}=7 \mathrm{BW}=250)$
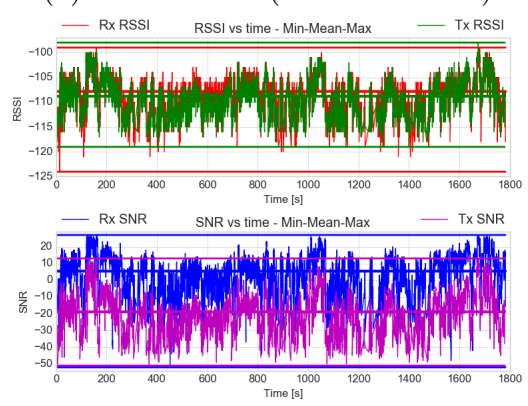

(d) RSSI \& SNR ( $\mathrm{SF}=8 \mathrm{BW}=250)$
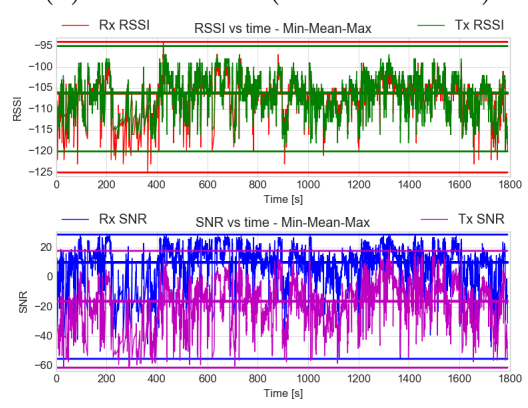

(f) RSSI \& SNR $(\mathrm{SF}=9 \mathrm{BW}=250)$
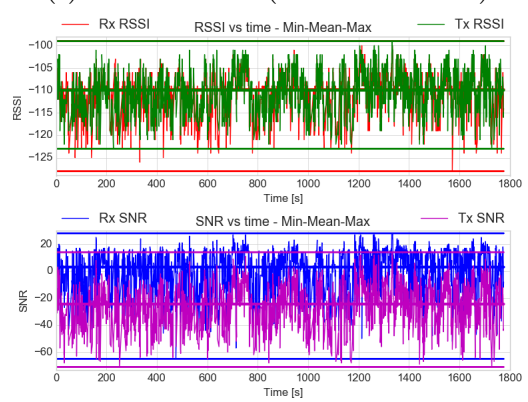

(h) RSSI \& SNR $(\mathrm{SF}=10 \mathrm{BW}=250)$ 

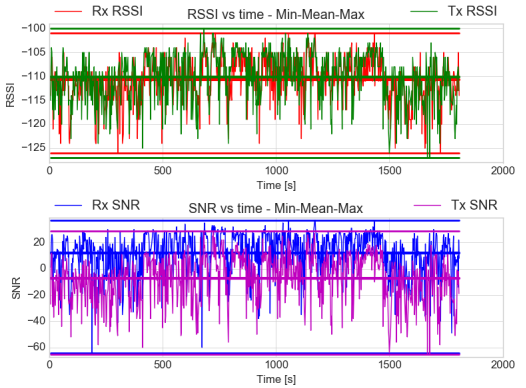

(i) RSSI \& SNR ( $\mathrm{SF}=11 \mathrm{BW}=125)$
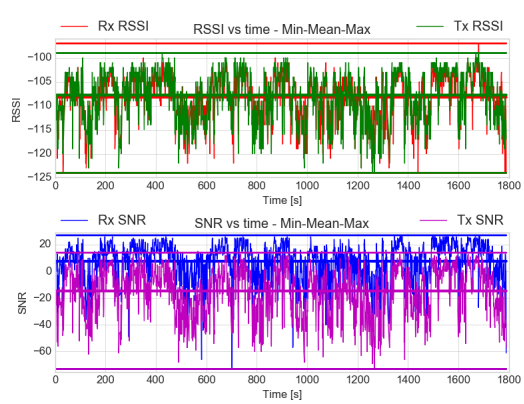

(j) RSSI \& SNR $(\mathrm{SF}=11 \mathrm{BW}=250)$

Fig. A: RSSI \& SNR values over time for Transmitter and Receiver

\section{B High density urban area}
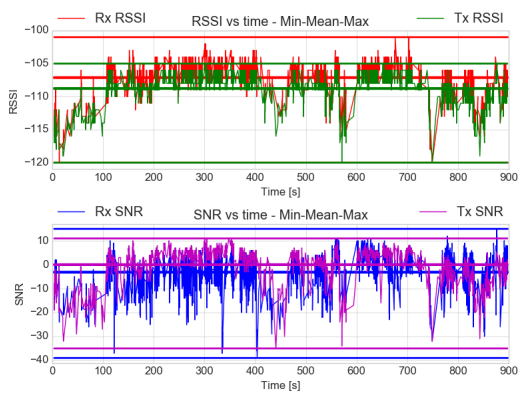

(a) RSSI \& SNR ( $\mathrm{SF}=7 \mathrm{BW}=125)$
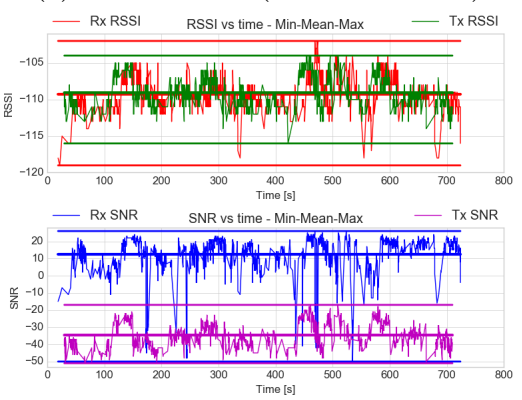

(c) RSSI \& SNR $(\mathrm{SF}=8 \mathrm{BW}=125)$
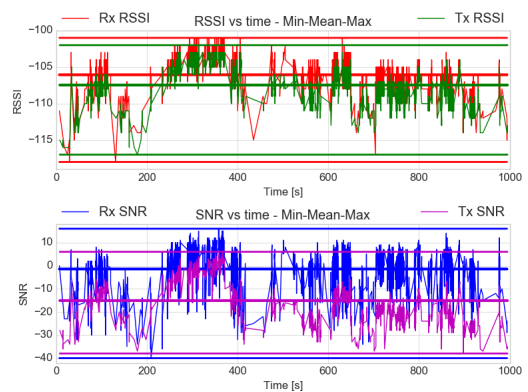

(b) RSSI \& SNR $(\mathrm{SF}=7 \mathrm{BW}=250)$
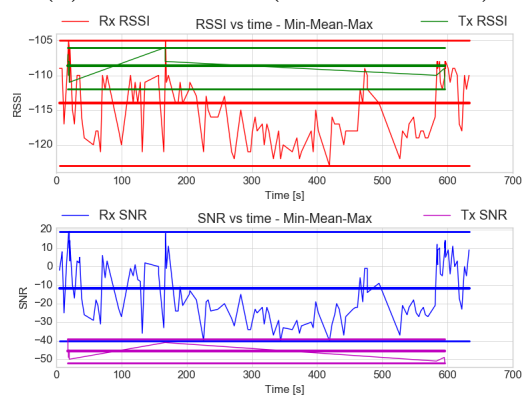

(d) RSSI \& SNR $(\mathrm{SF}=8 \mathrm{BW}=250)$ 

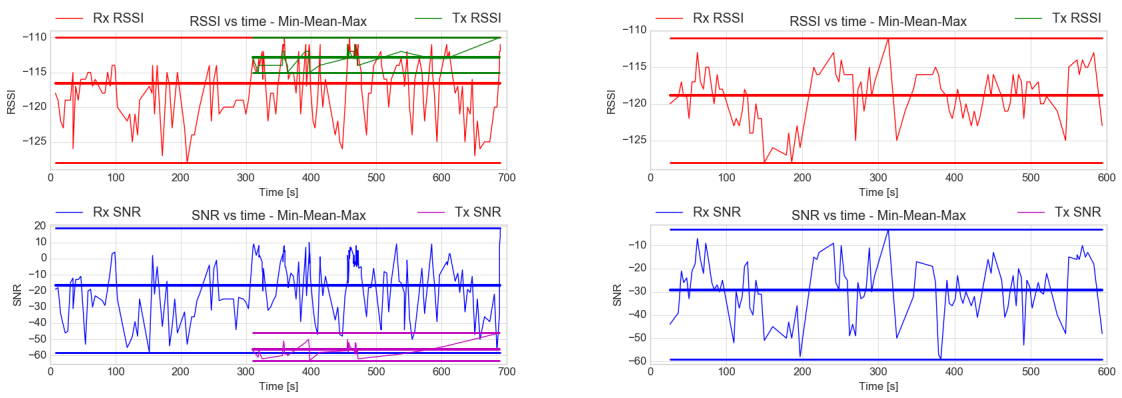

(e) RSSI \& SNR ( $\mathrm{SF}=9 \mathrm{BW}=125)$

(f) RSSI \& SNR $(\mathrm{SF}=9 \mathrm{BW}=250)$
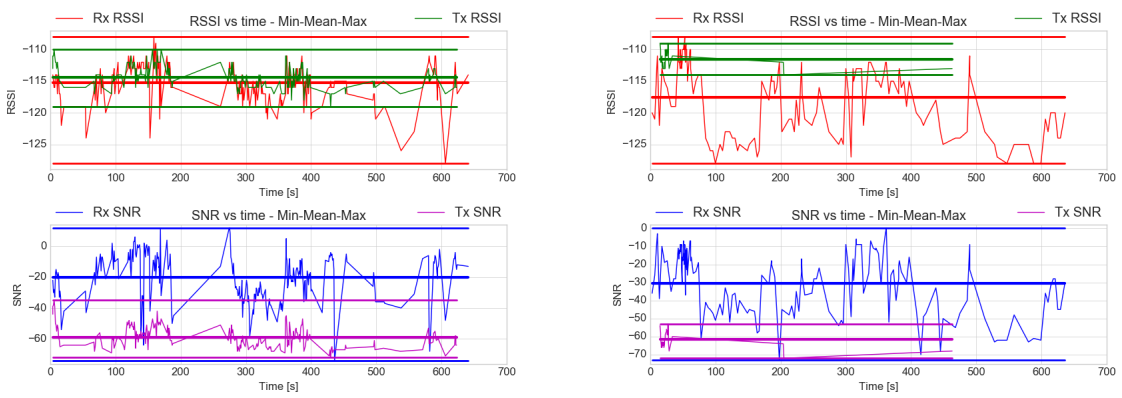

(g) RSSI \& SNR $(\mathrm{SF}=10 \mathrm{BW}=125)$

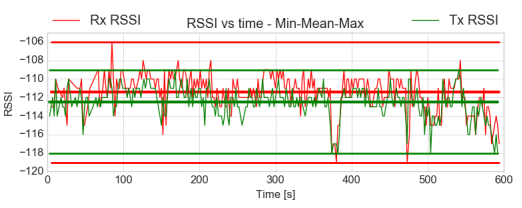

(h) RSSI \& SNR $(\mathrm{SF}=10 \mathrm{BW}=250)$

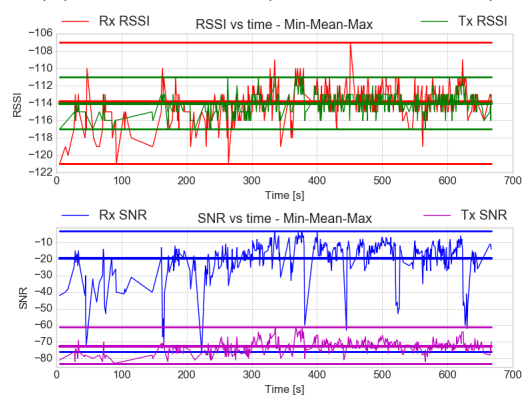

(i) RSSI \& SNR $(\mathrm{SF}=11 \mathrm{BW}=125)$

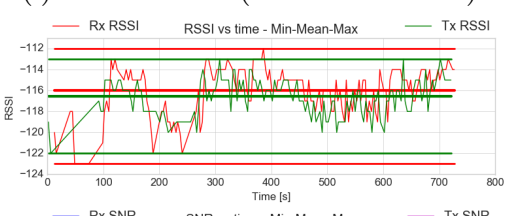

(j) RSSI \& SNR $(\mathrm{SF}=11 \mathrm{BW}=250)$
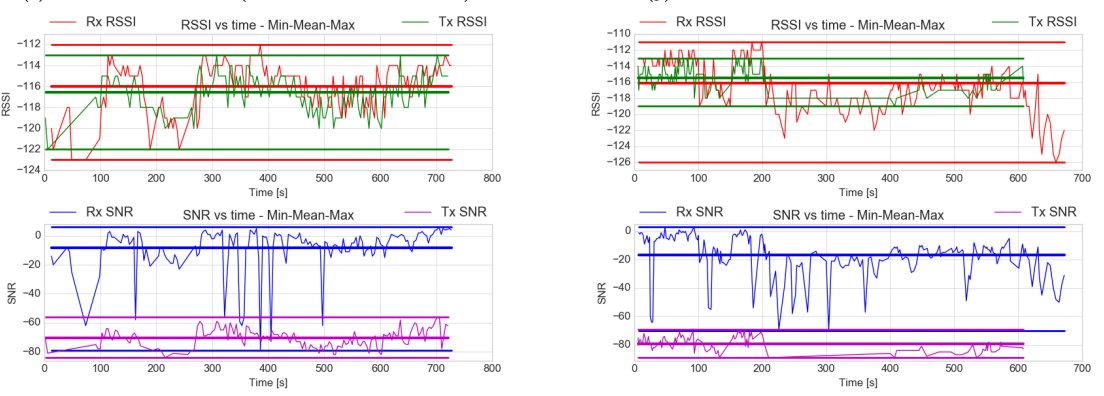

(k) RSSI \& SNR $(\mathrm{SF}=12 \mathrm{BW}=125)$

(l) RSSI \& SNR $(\mathrm{SF}=12 \mathrm{BW}=250)$

Fig. B: RSSI \& SNR values over time for Transmitter and Receiver 